\title{
The Effect of Altitude and Intensity of Physical Activity on the Exergy Efficiency of Respiratory System*
}

\author{
I.B. Henriques**1, C.E.K. $\mathrm{Mady}^{2}$, C. Albuquerque Neto ${ }^{3}$, J.I. Yanagihara ${ }^{4}$ and S. Oliveira Junior ${ }^{5}$ \\ ${ }^{1245}$ Polytechnic School of the University of São Paulo, Av. Prof. Mello Moraes, 2231, 05508-900, São Paulo, Brazil \\ ${ }^{3}$ Department of Mechanical Engineering, Centro Universitário da FEI, São Bernardo do Campo, Brazil \\ E-mail: ${ }^{1}$ izabela.henriques@usp.br, ${ }^{2}$ cekm@usp.br, ${ }^{3}$ cyroan@fei.edu.br, ${ }^{4}$ jiy @usp.br, ${ }^{5}$ soj@usp.br
}

\begin{abstract}
The effect of altitude on exercise performance of lowlanders has long been discussed, but it is still unclear whether the performance reduction is related to inefficiency of the respiratory system, body tissues or both. In the present work, exergy analysis was applied to the human body in order to compare its exergy efficiency under basal conditions and during physical activity at sea level and high altitudes for different periods of acclimatization. Two control volumes were analyzed: respiratory system and human body as a whole. Data concerning mass and energy balances of the body and respiratory system were obtained from models available in the literature, which were modified based on medical literature to simulate the responses to physical activity at high altitude for different periods of acclimatization. The results indicated that the respiratory system exergy efficiency is reduced at high altitudes and under physical activity, while exergy efficiency of the body increases for both parameters, which may indicate that the discomfort reported at high altitudes is mostly related to the respiratory system than to the other ones. Concerning the acclimatization period, its influence was more pronounced on the respiratory system.
\end{abstract}

\section{Keywords: Exergy analysis; exergy efficiency; respiratory system; altitude; physical activity}

\section{Introduction}

Concepts of the Second Law of Thermodynamics have first been formally applied to living organisms only in 1946, by Prigogine and Wiame [1]. They stated that living organisms tend to a state of minimum entropy production. By the end of the life cycle, the entropy production rate approaches its minimum value, while the total entropy of the organism reaches its maximum. The so-called Minimum Entropy Production Principle was confirmed by Zotin and Zotina [2], Balmer [3], Aoki [4], Silva and Annamalai [5, 6] and Mady et al. [7] for different species, including humans. Furthermore, Mady et al. [7] observed that exergy efficiency tends to decrease as a function of age, for basal conditions.

Batato et al. [8] proposed a pioneer model to perform exergy analysis of the human body. Later, Prek [9, 10], Prek and Butala [11] and Simone et al. [12] performed this kind of analysis to obtain relations between destroyed exergy, thermal comfort and thermal sensation conditions. Mady et al. [7] analyzed the destroyed exergy and exergy efficiency as a function of environmental conditions (operative temperature and relative humidity).

Another study concerning the exergy behavior of the human body was also conducted by Mady et al. [13]. Exergy analysis was applied in order to better understand the thermoregulatory system adopted in Ferreira and Yanagihara [14] thermal model of the human body. Destroyed exergy and exergy efficiency were calculated as a function of the constants of the control system of the model. They observed that the values attributed to the constants of the model, which were based on experiments, corresponded to the point of minimum destroyed exergy and maximum exergy efficiency.

A few authors applied exergy analysis $[15,16]$, or the Second Law analysis $[5,6,17]$ to the human body during physical exercise. Results of Rahman [17] indicate that the entropy generation rate increases with the increment of exercise level. For Mady et al. [15], the minimum entropy production principle is valid for people running, but exergy efficiency increases as a function of aging (different from the results for basal conditions)

Albuquerque-Neto et al. [16] made the first attempt to apply exergy analysis to respiratory system under physical activity. They evaluated exergy destruction in lungs and in tissues, and concluded that tissues are more effective than lungs during physical exercise. Some assumptions were made in their model: metabolism as a heat source, instead of a chemical reaction; air composed only of oxygen and carbon dioxide and reference state was assumed at the tissues.

Although the entropy production and destroyed exergy were already calculated for the whole body $[5,6,15,17]$ and for the lung [16] during physical activities, the exergy efficiency of this organ has not yet been discussed. In the present work, destroyed exergy and exergy efficiency are calculated as a function of altitude and intensity of physical activity. Acclimatization periods are also taken into account by means of hemoglobin concentration. Two models are employed to supply the basic data to the exergy analyses: Ferreira and Yanagihara [14] thermal model of the human body and Albuquerque, Yanagihara and Turri [18] model of the respiratory system. To evaluate the exergy performance of the body as a whole, the model of Mady et al. [7] and

\footnotetext{
* This paper is an updated version of a paper published in ECOS'12 proceedings. It is printed here with the permission of authors and organizers
} 
Mady and Oliveira Junior [19] are adopted. For the exergy analysis of the respiratory system, a model based on Albuquerque-Neto et al. [16] is proposed.

\section{Methods}

\subsection{Human body}

According to Mady et al. [7], exergy interactions between the body and the environment are caused by exergy flows due to respiration (inspired and expired air) and evaporation, exergy transfer associated with convection and radiation, and work performed by the body, as can be seen in Figure 1. The thermal model of Ferreira and Yanagihara [14] provides the necessary data to fulfill the analysis.

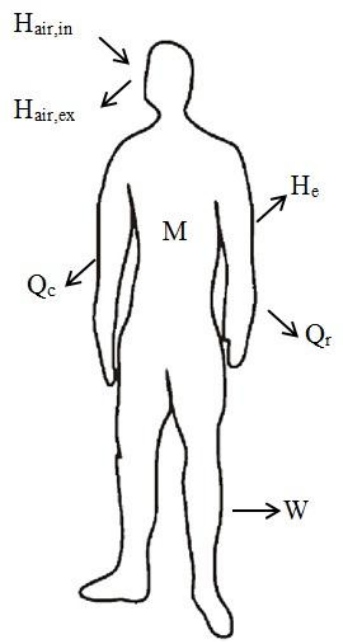

Figure 1: Interactions between the human body and environment. Adapted from Rahman [17].

A general exergy balance is obtained from the following equation:

$$
\frac{d \mathbf{B}}{d t}=\sum B_{\text {in }}-\sum B_{\text {out }}+\sum_{k} Q_{k}\left(1-\frac{T_{0}}{T_{k}}\right)-W-B_{d}
$$

For the human body,

$$
\frac{d \mathbf{B}_{b o d y}}{d t}=B_{a i r, i n}-B_{a i r, e x}-B_{c}-B_{r}-B_{e}-W-B_{d, b o d y}
$$

Exergy variation over time is divided into two parcels, namely exergy metabolism rate and exergy variation due to the variation in environmental conditions.

$$
\frac{d \mathbf{B}_{b o d y}}{d t}=-B_{M, b o d y}+\left.\frac{d \mathbf{B}_{b o d y}}{d t}\right|_{\Delta T}
$$

Rearranging Eqs. (2) and (3), the rate of exergy destroyed in the body is:

$$
B_{d, b o d y}=B_{M, b o d y}-\left(B_{c}+B_{r}+B_{e}+\Delta B_{a i r}\right)-\left.\frac{d \mathbf{B}_{b o d y}}{d t}\right|_{\Delta T}-W
$$

The general exergy efficiency of the body is determined by Eq. (5), where the last term represents the ratio of destroyed exergy to the exergy provided, which is composed of exergy metabolism, exergy variation due to changes in temperature and exergy flow rate that enters the control volume, in this case, exergy of inspired air:

$$
\eta_{b o d y}=1-\frac{B_{d, b o d y}}{B_{M, b o d y}-d \mathbf{B}_{b o d y} /\left.d t\right|_{\Delta T}+B_{a i r, \text { in }}}
$$

Metabolism is the energy released to the body by oxidation of the nutrients ingested. Its exergy parcel is associated with the chemical exergy of substrates and the stoichiometric coefficients of oxidation reactions. Mady and Oliveira-Junior [19] assumed that glucose, palmitic acid and an average amino acid correspond, respectively, to carbohydrates, lipids and proteins ingested. Exergy metabolism as a function of mass flow rates of oxygen consumption, carbon dioxide production and nitrogen excretion through urine is calculated as:

$$
B_{M, b o d y}=9558 m_{O_{2}, b o d y}+3928 m_{\mathrm{CO}_{2}, \text { body }}-456 m_{N, b o d y}
$$

The power performed by the body is calculated from external performed work defined by Cavagna [20], as a function of mass and speed.

\subsection{Respiratory system}

A model of the respiratory system comprising heat transfer, blood flow and gas exchange was developed by Albuquerque et al. [16]. According to their model, inspired air enters the lungs through the airways and flows to the alveolar compartment, which interfaces with pulmonary capillaries compartments. Meanwhile, blood coming from the venous compartment, which carries the carbon dioxide produced by tissues metabolism, goes through the capillaries. A small fraction of venous blood, 2\% approximately, does not flow to the capillaries, being mixed directly to arterial blood as indicated in Figure 2. Due to the difference between gases partial pressures in alveolar compartment and capillaries, oxygen diffuses to capillaries, while carbon dioxide flows to alveolar compartments, turning venous into arterial blood, which goes to the arterial compartment before going through the tissues. Carbon dioxide is expired to the environment. The same gas exchange process takes place in the tissues, but with opposite direction, providing oxygen to metabolic reactions of tissue compartments.

In order to perform the exergy analysis of the respiratory system, the boundary of the control volume was defined around the lungs and airways, which differs from Albuquerque-Neto et al. [16]. Figure 3 indicates the inspired air and venous blood that enter the control volume and the expired air and arterial blood that leave it. Internal energy variation due to metabolism, as well as the metabolic heat transfer to the rest of the trunk by conduction, is also considered. Moreover, the work performed in order to contract and to expand the lungs, which is called work of breathing, is added to the system. 


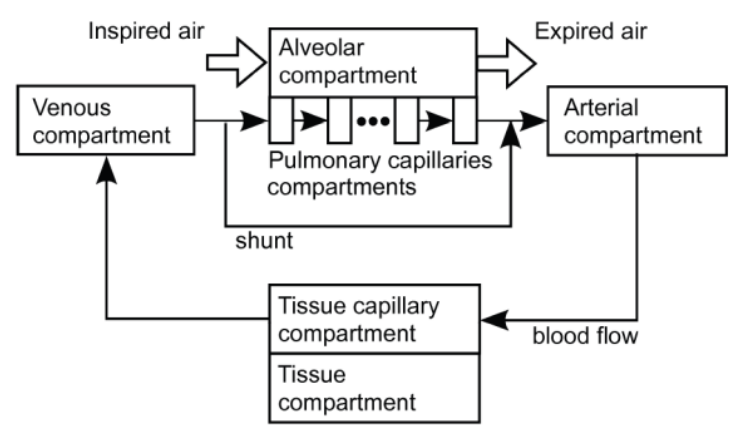

Figure 2: Schematic representation of the model proposed by Albuquerque et al. [16].

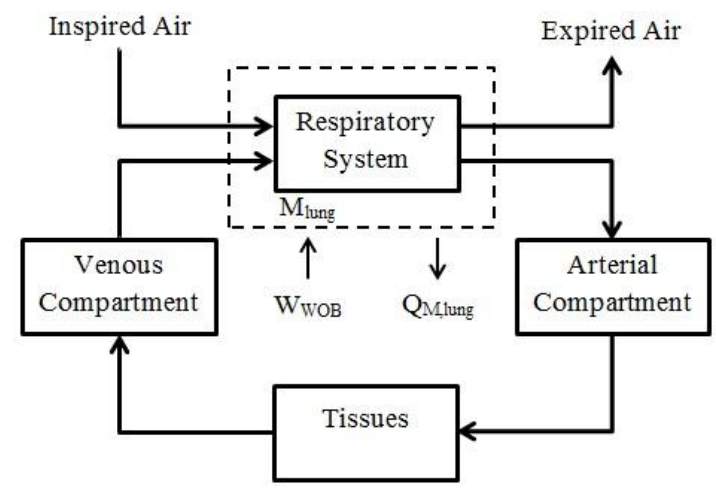

Figure 3: Representation of the adopted control volume.

On an exergy basis, the components of the balance correspond to exergy flows rates associated with venous blood and inspired air in the inlet, arterial blood and expired air in the outlet, internal exergy variation rate due to exergy metabolism of the lung, an exergy transfer caused by the metabolism of the lung and the power of breathing, which is applied to the system by the surroundings. Thus,

$$
\begin{aligned}
& B_{d, \text { lung }}=B_{M, \text { lung }}+B_{b l, v e}+B_{\text {air }, \text { in }}+W_{W O B}-B_{b l, a r} \\
& -B_{\text {air }, \text { ex }}-Q_{M, \text { lung }}\left(1-\frac{T_{0}}{T_{\text {lung }}}\right)-\left.\frac{d \mathbf{B}_{\text {lung }}}{d t}\right|_{\Delta T}
\end{aligned}
$$

Mass flow rates of oxygen produced and carbon dioxide consumed by lung tissues, as well as the nitrogen excreted, are used to determine its exergy $\left(B_{M, \text { lung }}\right)$ and energy metabolism $\left(M_{\text {lung }}\right)$ rates. The former is obtained by Eq. (8), and the latter by Eq. (9).

$$
\begin{aligned}
& B_{M, \text { lung }}=9558 m_{\mathrm{O}_{2}, \text { lung }}+3928 m_{\mathrm{CO}_{2}, \text { lung }}-456 m_{N, \text { lung }} \\
& M_{\text {lung }}=1179 m_{\mathrm{O}_{2}, \text { lung }}+2502 m_{\mathrm{CO}_{2}, \text { lung }}-129 m_{N, \text { lung }}
\end{aligned}
$$

The air is assumed to be an ideal gas composed of oxygen, carbon dioxide, nitrogen and water vapor. Therefore, its exergy is the sum of the exergy of its components:

$$
B_{\text {air }}=B_{\mathrm{O}_{2}}+B_{\mathrm{CO}_{2}}+B_{\mathrm{N}_{2}}+B_{w}
$$

The exergy of an ideal gas is a function of its mass flow rate, specific heat capacity, temperature, gas constant and partial pressure, as well as the reference state. In Eq. (11) the subscript $g$ refers to each gas that composes the air.

$B_{g}=m_{g}\left[c_{p, g}\left(T_{a i r}-T_{0}-T_{0} \ln \frac{T_{a i r}}{T_{0}}\right)+R_{g} T_{0} \ln \frac{P_{g, a i r}}{P_{g, 0}}\right](11)$

Bearing in mind that the reference state is the environment, air properties of inspired air are equal to the reference state. Thus, exergy of inspired air is zero. For expired air:

$$
\begin{aligned}
& B_{\text {air }, e x}=B_{O_{2}, e x}+B_{C O_{2}, e x}+B_{N_{2}, e x}+B_{w, e x} \\
& B_{g, e x}=m_{g, e x}\left[c_{p, g}\left(T_{e x}-T_{0}-T_{0} \ln \frac{T_{e x}}{T_{0}}\right)+R_{g} T_{0} \ln \frac{P_{g, e x}}{P_{g, 0}}\right]
\end{aligned}
$$

Blood was analyzed as a mixture of incompressible liquid and ideal gases, in this case, oxygen and carbon dioxide, as long as the nitrogen does not diffuse through the capillaries towards bloodstream. Thus,

$$
B_{b l}=B_{l i q}+B_{O_{2}}+B_{C_{2}}
$$

where

$$
B_{l i q}=m_{b l}\left[c_{b l}\left(T_{b l}-T_{0}-T_{0} \ln \frac{T_{b l}}{T_{0}}\right)\right]
$$

Exergy of venous blood flow is obtained as:

$$
B_{b l, v e}=B_{l i q, v e}+B_{O_{2}, v e}+B_{C_{2}, v e}
$$

$$
B_{l i q, v e}=m_{b l, v e}\left[c_{b l}\left(T_{b l, v e}-T_{0}-T_{0} \ln \frac{T_{b l, v e}}{T_{0}}\right)\right]
$$

$$
B_{g, v e}=m_{g, v e}\left[c_{p, g}\left(T_{b l, v e}-T_{0}-T_{0} \ln \frac{T_{b l, v e}}{T_{0}}\right)+R_{g} T_{0} \ln \frac{P_{g, v e}}{P_{g, 0}}\right]
$$

For arterial blood:

$$
B_{b l, a r}=B_{l i q, a r}+B_{O_{2}, a r}+B_{C_{2}, a r}
$$

Each parcel is determined for arterial blood in the same way as for the venous one, by substituting the corresponding terms of arterial blood in Eq. (17) and (18). 
The work of breathing was obtained from the study developed by Fritts, Filler and Fishman [21], which analyzed how the power of breathing was influenced by ventilation. Pressure-volume curves of the lung were plotted for different subjects and different values of ventilation. Power of breathing was determined by integrating the pressure-volume curves.

Considering that $W_{W O B}$ is an external work performed by the surrounding on the control volume, the heat transferred to the body due to the lung metabolism is:

$Q_{M, l u n g}=M_{\text {lung }}+W_{W O B}$

Eq. (21) determines the exergy efficiency of the respiratory system. As well as the exergy efficiency of the body (Eq. (5)), the last term represents the ratio of destroyed exergy to exergy provided. The exergy provided to this control volume is the sum of the exergy metabolism of the lung, its exergy variation over time due to temperature variations, work of breathing, and exergy flows that enter the control volume: exergy of venous bloodstream and exergy of inspired air.

$$
\eta_{\text {lung }}=1-\frac{B_{d, \text { lung }}}{B_{M, \text { lung }}-d \mathbf{B}_{\text {lung }} /\left.d t\right|_{\Delta T}+W_{W O B}+B_{b l, v e}+B_{\text {air }, \text { in }}}
$$

In the present work, environmental temperature is assumed to be constant. Moreover, despite being calculated over time, exergy rates are indicated only for steady state condition. Therefore, time related terms become zero and, substituting $B_{\text {d,lung }}$ for Eq. (7):

$$
\eta_{\text {lung }}=\frac{B_{b l, a r}+B_{a i r, e x}+B_{Q_{M}, \text { lung }}}{B_{M, l u n g}+W_{W O B}+B_{b l, v e}+B_{a i r, i n}}
$$

\subsection{Simulations}

To better analyze the influence of the parameters in exergy efficiency, fifty one simulations were run. For each altitude $(0,1500,3000$ and $4500 \mathrm{~m})$, three intensities of physical activity were tested, and for each physical activity level, different periods of acclimatization were evaluated, except for sea level, since the study is applied to lowlanders. In addition, scenarios where the subject acclimatized to $4500 \mathrm{~m}$ returns to the other three altitude levels were also simulated. In order to emphasize the role played by those parameters, temperature and relative humidity were kept constant at $20^{\circ} \mathrm{C}$ and $50 \%$, respectively.

With regard to the inputs of the models, altitude is expressed as the corresponding atmospheric pressure, intensity of physical activity is represented by metabolism and acclimatization period is related to hemoglobin concentration. The relation between atmospheric pressure and altitude measured in $\mathrm{kPa}$ and meters, respectively, is determined by Eq. (24) [22].

$P_{0}=101.3\left(\frac{288-0.0065 \cdot A}{288}\right)^{5.257}$
For each exercise intensity, standard energy metabolism of the body is set as an input of the model and its values are obtained from ASHRAE [23].

Bärtsch \& Saltin [24] synthesized the effects of the acclimatization period on some physiological parameters. Among the parameters analyzed, the only one that is an input of the Albuquerque-Neto et al. [16] model is hemoglobin concentration. According to them, when a lowlander gets to a place located above $500 \mathrm{~m}$, without any previous adaptation period, hemoglobin concentration decreases during the first two days, assuming its lowest value by this last moment. From the third day up to the third week, hemoglobin concentration experiences a pronounced increase. For altitudes up to $1500 \mathrm{~m}$, this value remains constant, whereas it still increases slightly for altitudes above this limit.

\section{Results and Discussion}

Destroyed exergy and exergy efficiency were calculated for body and lungs as a function of altitude, exercise intensity and acclimatization periods. Results related to exergy destroyed by the body and the lungs are presented in Figure 4. As can be seen, both parameters increase as the intensity of physical activity is incremented. For the same altitude, the increase observed between rest and walk for $B_{d, \text { body }}$ and $B_{d, \text { lung }}$ are, respectively, $122.8 \%$ and $158.5 \%$, while increments from walking to running are $25.5 \%$ and $50.0 \%$. However, $B_{d, \text { body }}$ and $B_{d, \text { lung }}$ have opposite behaviors as far as altitude is concerned. Regarding $B_{d, b o d y}$, it is possible to observe that a direct relation to altitude is clearer under physical activity than at rest. Analyzing the individual parcels that compose $B_{d, b o d y}$ at rest, it is possible to observe that exergy metabolism at $4500 \mathrm{~m}$ does not follow the decreasing trend observed from $0 \mathrm{~m}$ to $3000 \mathrm{~m}$, being greater instead. $B_{M, b o d y}$ growth is a consequence of activating shivering mechanism, which is an indirect consequence of reductions of the coefficient of heat transfer by convection and oxygen partial pressure that take place at high altitudes. The first causes an augmentation of skin temperature, which, combined with the latter, stimulates blood circulation, reducing internal temperature, finally leading to the activation of the shivering metabolism and consequent augmentation of exergy metabolism and exergy destroyed in the body. This behavior is observed only at rest due to the lower basal metabolism in comparison with the metabolism under activity. During physical exercise, the higher the altitude, the smaller the exergy destroyed by the body. Meanwhile, the opposite happens to the respiratory system, even at rest: the exergy destroyed by the lungs increases as altitude gets higher. About the acclimatization period, its effects are more pronounced on $B_{d, l u n g}$ than on $B_{d, b o d y}$. During the first two days of altitude exposure, $B_{d, \text { lung }}$ experiences a slight growth, around $2 \%$. From the second day on, it begins to decrease, reaching its lowest value, which is $4.8 \%$ smaller than the one without acclimatization, and stabilizing around the twentieth day. 

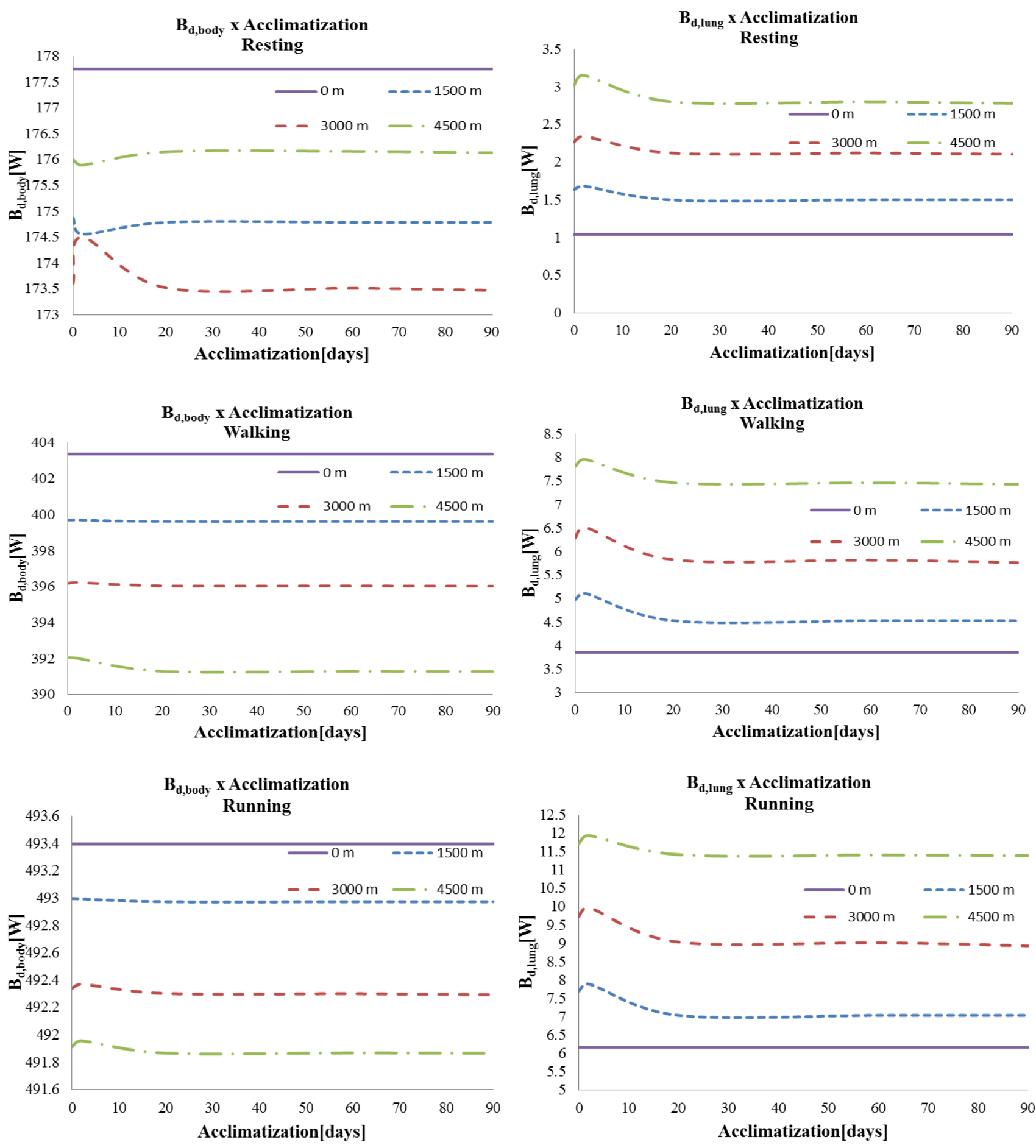

Figure 4: Exergy destroyed by the body $\left(B_{d, b o d y}\right)$ and the lung $\left(B_{d, l u n g}\right)$ as a function of altitude and acclimatization period for different physical activity intensities.

Regarding the parcels that composed $B_{d, l u n g}$, the behavior of $\Delta B_{\text {res }}$, which is the difference between the exergy of expired and inspired air, is noteworthy. As can be seen in Figure 5 , the $\Delta B_{\text {res }}$ value does not vary with the altitude, which is interesting, taking into account that partial pressures of gases in expired air and environment, which is the reference state, vary. The respiratory system could be analyzed as a blood treatment plant, where $\Delta B_{\text {res }}$ would correspond to the waste of the process. Thus, $\Delta B_{\text {res }}$ behavior indicates that the exergy of the waste of respiratory process

is kept constant despite oxygen availability in the environment, which may indicate that the mechanisms associated to respiration control are activated based on exergy responses in order to keep the difference between exergy of expired and inspired air constant for each activity intensity, assuring the capture of oxygen available in the air and the removal of carbon dioxide in venous blood. This fact, in addition to the results in Mady et al. [13] concerning the constants of the thermoregulatory system, indicates that control mechanisms of human body may be associated with exergy parameters. 


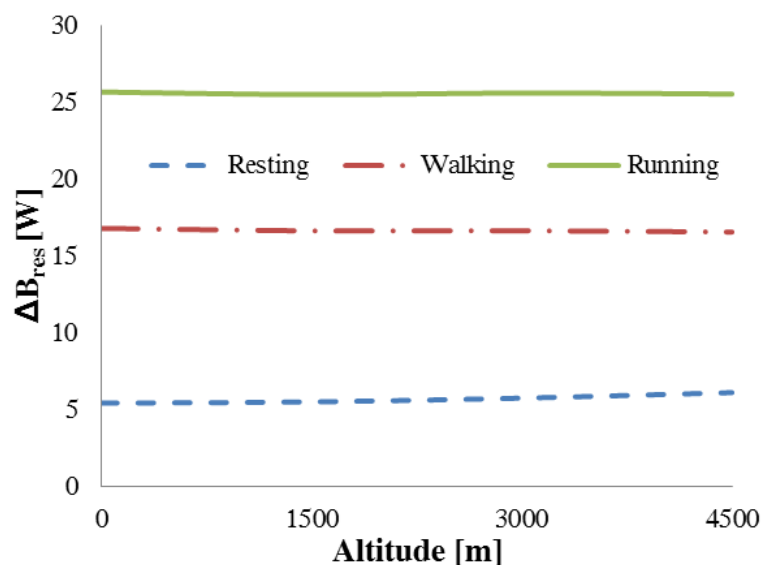

Figure 5: Difference between exergy of expired and inspired air $\left(\Delta B_{\text {res }}\right)$ as a function of altitude for different physical activity intensities.

Additionally, in order to emphasize the impact of the exergy destroyed in the lung on total exergy destroyed in the body for the different scenarios studied, the ratio of $B_{d, \text { lung }}$ to $B_{d, b o d y}$ was determined and the results are shown in Figure 6 . The graphs evidence that, at higher altitudes and exercise intensities, the contribution of $B_{d, \text { lung }}$ to total $B_{d, b o d y}$ increases.

Figure 7 shows the results for exergy efficiency of body and lungs for all scenarios. As the altitude increases, $\eta_{\text {lung }}$ decreases, whereas $\eta_{\text {body }}$ tends to increase, except for $4500 \mathrm{~m}$ at rest, in agreement with $B_{d, b o d y}$ and $B_{M}$ behavior. This result indicates that, at high altitudes, other organs of the body must improve their exergy efficiencies in order to compensate the decline of lung efficiency, which is coherent with the results for the destroyed exergy. In turn, the growth of $B_{d, b o d y}$ with the physical activity intensity is also followed by a growth of $\eta_{b o d y}$, whose values are $197.4 \%$ and $42.2 \%$ from resting to walking and walking to running, respectively. This information demonstrates that, under physical activity, the increase of exergy metabolism of the body overcomes the augmentation of its destroyed exergy. As a result, greater exergy efficiencies are obtained. Regarding the lung, exergy efficiency is reduced, for both altitude and exercise, accompanying $B_{d, \text { lung }}$ increase, but in a much lower percentage. Exergy efficiency reduction is about $0.2 \%$ between each altitude. With respect to physical activity, $\eta_{\text {lung }}$ declines at a $0.25 \%$ average between resting and walking. The reduction for the next activity level is $0.07 \%$. This small variation, as well as high $\eta_{\text {lung }}$ values, is related to the nature of the processes that take place in the respiratory system, which is essentially a mass exchanger. According to Mady and Oliveira-Junior [19], most of the irreversibility generated in the body is associated with the metabolism. For the lung, on an exergy basis, $B_{M \text {,lung }}$ corresponds to only $0.3 \%$ of the total exergy provided, while it represents $70 \%$ of $B_{d, l u n g}$, which explains its high exergy efficiency. About the adaptation period, it is clear that the effect of acclimatization is more evident for the lung than for the body, confirming the information provided by destroyed exergy data.
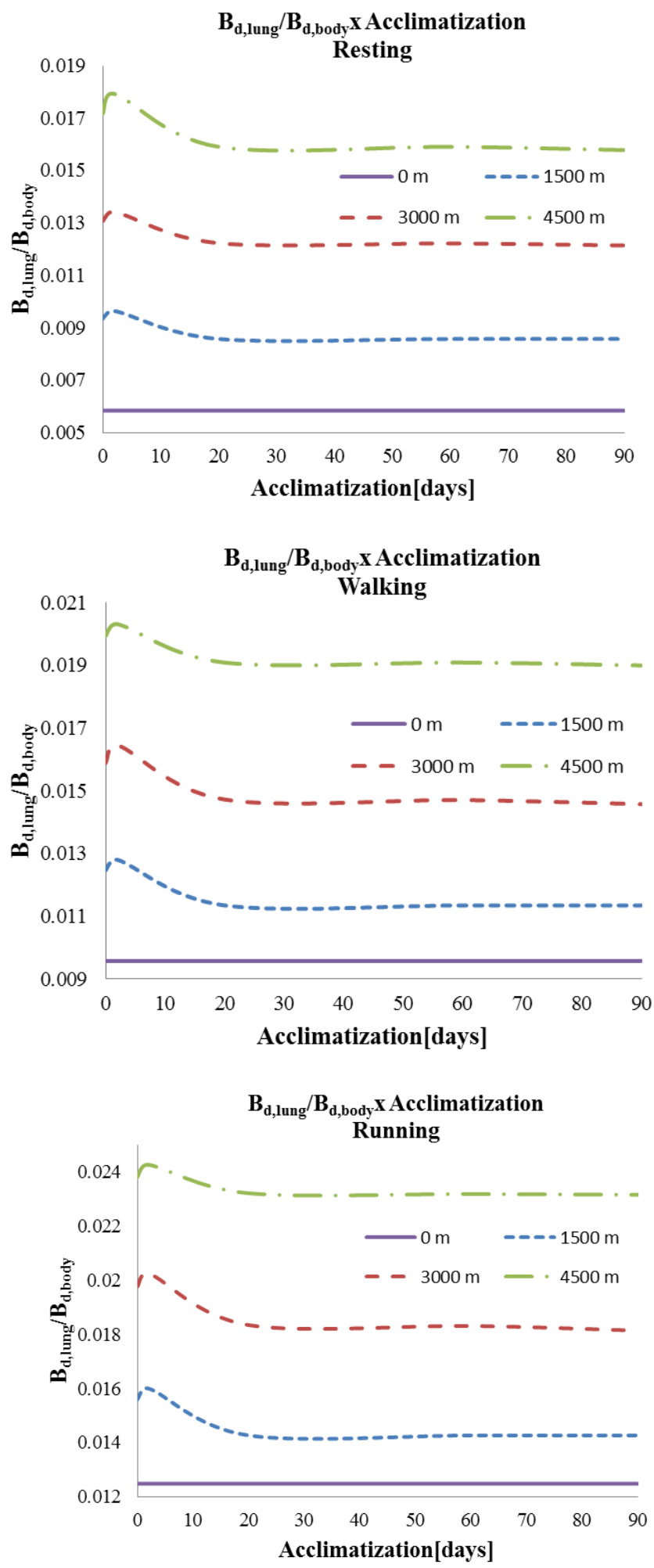

Figure 6: Comparative of the ratio of the exergy destroyed by the lung $\left(B_{d, \text { lung }}\right)$ to the exergy destroyed by the body $\left(B_{d, b o d y}\right)$ as a function of altitude and acclimatization period for different physical activity intensities. 

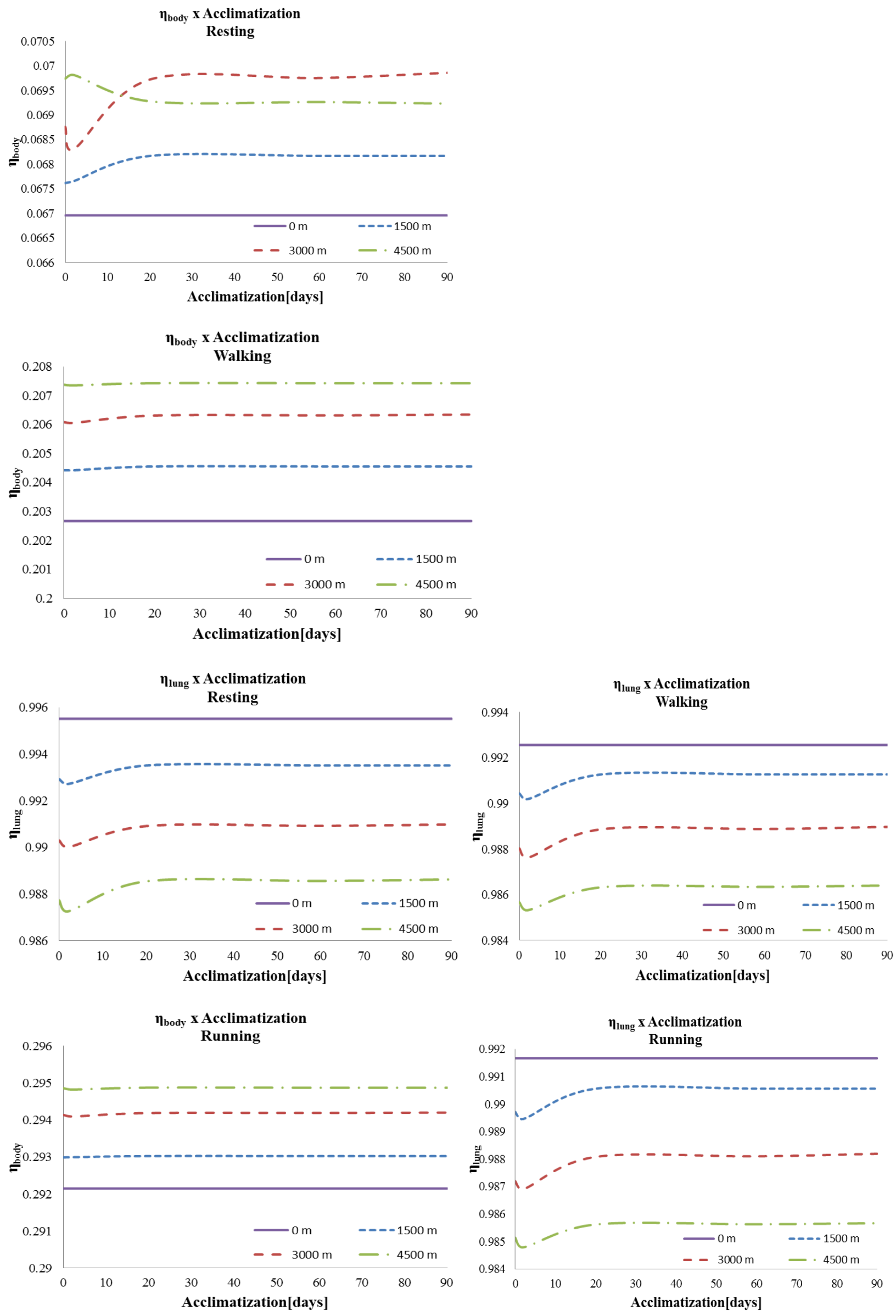

Figure 7: Exergy efficiency of the body $\left(\eta_{\text {body }}\right)$ and the lung $\left(\eta_{\text {lung }}\right)$ as a function of altitude and acclimatization period for different physical activity intensities. 
Furthermore, data regarding exergy efficiencies in the situation in which the subject returns to the other three altitude levels after ninety days of acclimatization at 4500 $\mathrm{m}$ were also attained and are displayed in Figure 8, together with the results for a non-acclimatized subject. A positive effect of the acclimatization period can be seen in both $\eta_{b o d y}$ and $\eta_{\text {lung. }}$. Yet, the more intense the exercise, the smaller the difference between the exergy efficiency of the body for acclimatized and non-acclimatized, while this difference is more pronounced for the lung. Moreover, the effect of acclimatization is more pronounced when the subject returns to lower altitudes.
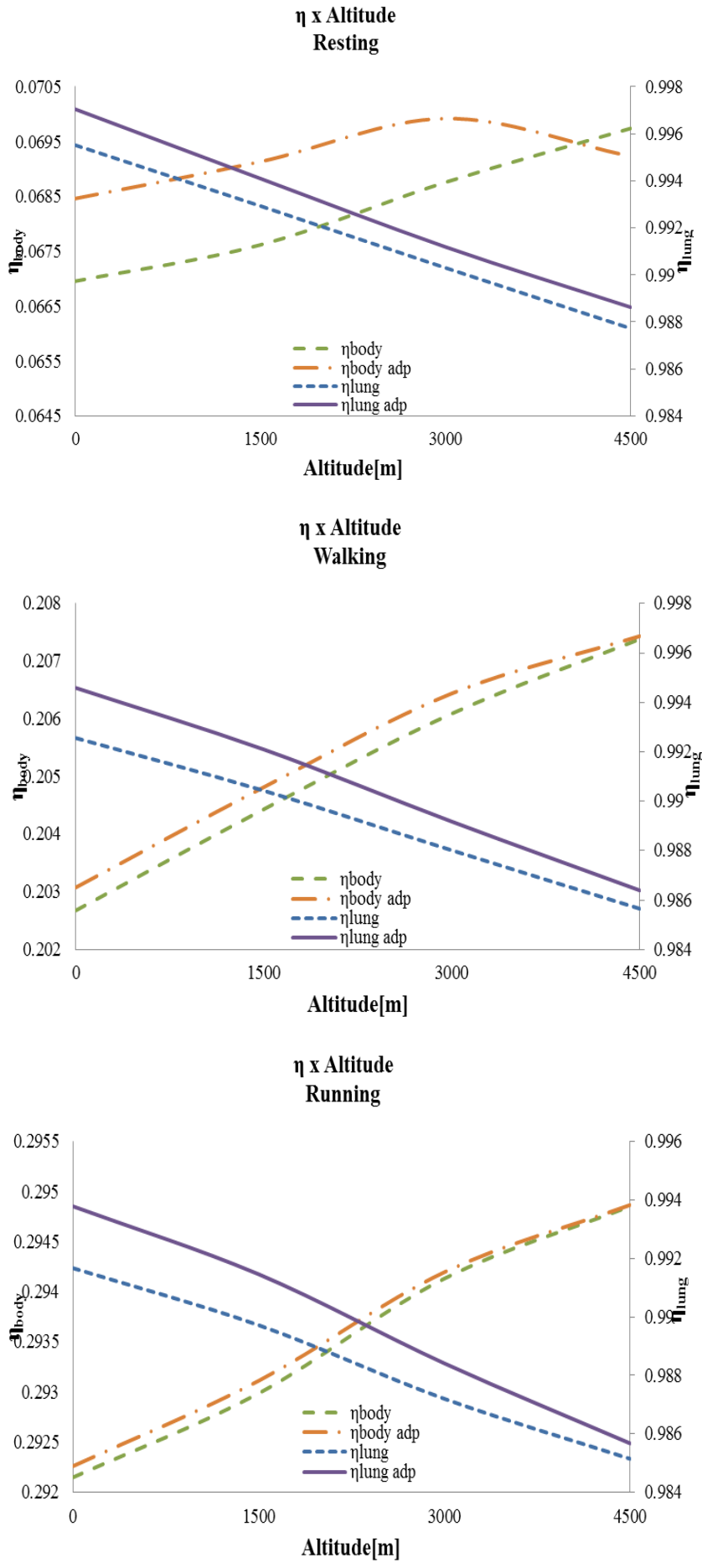

Figure 8: Exergy efficiency of the body $\left(\eta_{\text {body }}\right)$ and the lung $\left(\eta_{\text {lung }}\right)$ before and after ninety days of acclimatization at $4500 \mathrm{~m}$ as a function of the current altitude.

\section{Conclusions}

Destroyed exergy and exergy efficiency were determined for body and lungs at different altitudes, physical activity intensities and acclimatization periods. From the results, different patterns of behavior were observed between the body and the respiratory system concerning altitude and exercise intensity. Both $B_{d, b o d y}$ and $B_{\text {d,lung }}$ increases as physical activity intensity is enhanced. However, regarding altitude, the exergy destroyed in the body decreases with its increment, while exergy destroyed in the lung increases. Investigating the individual exergy components of $B_{d, l u n g}$, it was observed that $\Delta B_{\text {res }}$, which may be taken as the waste of the respiratory process, is kept constant, despite altitude augmentation. This information supports the idea that the operation of control systems of the body is related to exergy parameters. Moreover, the higher the altitude and activity intensity, the greater the contribution of $B_{d, l u n g}$ to $B_{d, b o d y}$. In accordance with the results about destroyed exergy, as the altitude is incremented, the body exergy efficiency increases, while the lung exergy efficiency becomes lower. However, due to a marked increase of exergy metabolism of the body, $\eta_{b o d y}$ follows the exercise augmentation, despite the increase of $B_{d, b o d y}$. Meanwhile, $\eta_{\text {lung }}$ is negatively affected by physical activity. Concerning the acclimatization, $\eta_{\text {lung }}$ is more affected than $\eta_{b o d y}$, and its highest values are obtained after twenty days of acclimatization. Besides, both $\eta_{\text {body }}$ and $\eta_{\text {lung }}$ increase for the acclimatized subject at lower altitudes.

Finally, the discomfort felt under physical activity at high altitudes seems to be associated with the respiratory system, since exergy efficiency of the body increases at both altitude and activity, while the opposite happens to the lung, indicating that the exergy performance of the other organs and systems is improved under these conditions. Moreover, the results indicate a relation between the exergy behavior of the human body and its regulatory systems, which endorses the application of exergy analysis as a tool to better understand the physiological mechanisms whose operations are still unclear.

\section{Acknowledgements}

Authors 1, 2 and 4 acknowledge $\mathrm{CNPq}$ (National Research Council) and FAPESP (the State of São Paulo Research Foundation) for the financial support.

\section{Nomenclature}

A altitude, $\mathrm{m}$

B exergy, $\mathrm{J}$

$B \quad$ exergy rate and flow rate, $\mathrm{W}$

$c \quad$ specific heat capacity, $\mathrm{J} /(\mathrm{kg} . \mathrm{K})$

$H \quad$ enthalpy flow rate, $\mathrm{W}$

$M$ metabolism, W

$m$ mass flow rate, $\mathrm{kg} / \mathrm{s}$

$Q \quad$ heat transfer rate, W

$P$ pressure, $\mathrm{Pa}$

$R \quad$ gas constant, $\mathrm{J} /(\mathrm{K} . \mathrm{kg})$

$T$ temperature, ${ }^{\circ} \mathrm{C}$ or $\mathrm{K}$

$t \quad$ time, $\mathrm{s}$

$W \quad$ performed power, $\mathrm{W}$

\section{Greek symbols}

$\eta \quad$ efficiency

Subscripts and superscripts

0 reference 


$\begin{array}{ll}\text { ar } & \text { arterial } \\ \text { body } & \text { body } \\ \text { bl } & \text { blood } \\ \text { c } & \text { convective } \\ \text { dest } & \text { destroyed } \\ \text { e } & \text { evaporative } \\ \text { ex } & \text { expired } \\ \mathrm{g} & \text { gas } \\ \text { in } & \text { inflow } \\ \text { liq } & \text { liquid } \\ \mathrm{M} & \text { metabolic } \\ \text { out } & \text { outflow } \\ \mathrm{r} & \text { radiative } \\ \text { resp } & \text { respiration } \\ \Delta \mathrm{T} & \text { due to temperature variation } \\ \text { ve } & \text { venous } \\ \text { WOB } & \text { work of breathing }\end{array}$

\section{References}

[1] Prigogine, I., Wiame, J. Biologie et Thermodynamique des phenomenes irreversibles. Experimentia, 2(11):451453, 1964.

[2] Zotin, A. I., Zotina, R. S. Thermodynamics aspects of developmental biology. J. Theoretical Biology, 17,5775, 1967.

[3] Balmer, R. T. Entropy and aging in biological systems. Chemical Engineering Communications, 17, 171-181, 1984.

[4] Aoki, I. Entropy principle for human development, growth and aging. J. Theoretical Biology, 150, 215-223, 1991.

[5] Silva, C., Annamalai, K. Entropy generation and human aging: lifespan entropy and effect of Physical Activity Level. Entropy, 10,100-123, 2008.

[6] Silva, C., Annamalai, K. Entropy generation and human aging: lifespan entropy and effect of diet composition and caloric restriction diets. J. Thermodynamics; 2009:1-10, 2009.

[7] Mady, C. E. K., Ferreira, M. S., Yanagihara, J. I., Saldiva, P. H. N., Oliveira Junior, S. Modeling the exergy behavior of human body. Energy, 4, 546-553, 2012.

[8] Batato, M., Borel, L., Deriaz O., Jequier, E. Analyse exergétique théorique et expérimentale du corps humain. Entropie, 26, 120-130, 1990.

[9] Prek, M. Thermodynamic analysis of human heat and mass transfer and their impact on thermal comfort. Int. J. Heat and Mass Transfer,48, 731-739, 2005.

[10] Prek, M. Thermodynamical analysis of human thermal comfort. Energy, 31, 732-743, 2006.

[11] Prek, M., Butala, V. Principles of exergy analysis of human heat and mass exchange with the indoor environment. Int. J. Heat and Mass Transfer, 48, 731739, 2010.
[12] Simone, A., Kolarik, J., Iwamatsu, T., Asada, H., Dovjak, M., Schellen, L., Shukuya, M., Olesen, B.W. A relation between calculated human body exergy consumption rate and subjectively assessed thermal sensation. Energy and Buildings, 43, 1-9, 2011.

[13] Mady, C. E. K., Ferreira, M. S., Yanagihara, J. I., Saldiva, P. H. N., Oliveira Junior, S. Second law of thermodynamics and human body. Engenharia Térmica (Thermal Engineering), 10, 88-95, 2011.

[14] Ferreira, M. S., Yanagihara, J. I. A transient threedimensional heat transfer model of the human body. International Communications in Heat and Mass Transfer, 36, 718-724, 2009.

[15] Mady, C. E. K., Albuquerque Neto, C., Fernandes, T. L., Hernandez, A. J., Saldiva, P. H. N., Yanagihara, J. I., Oliveira-Junior, S. Exergy performance of human body during physical activities. Energy, 62, 370-378, 2013.

[16] Albuquerque-Neto, C., Pellegrini, L.F., Ferreira, M.S., Oliveira Jr, S., Yanagihara, J.I. Exergy analysis of human respiration under physical activity. Int. $J$. Thermodynamics, 13, 105-109, 2010.

[17] Rahman, A. A novel method for estimating the entropy generation rate in a human body. Thermal Science, 11, 75-92, 2007.

[18] Albuquerque, C.; Yanagihara, J. I., Turri, F. A carbon monoxide transport model of the human respiratory system applied to urban atmosphere exposure analysis. J. Brazilian Society of Mechanical Sciences and Engineering, 30, 253-260, 2008.

[19] Mady, C. E. K., Oliveira-Junior, S. Human body exergy metabolism. Int. J. Thermodynamics, 16, 73-80, 2013.

[20] Cavagna, G. A., Kaneko, M. Mechanical work and efficiency in level walking and running. J. Physiology, 68, 467-481, 1977.

[21] Fritts, H. W., Filler, Jr J., Fishman, A. P., Cournand, A. The efficiency of ventilation during voluntary hyperpnea: studies in normal subjects and in dyspneic patients with either chronic pulmonary emphysema or obesity. J. Clinical Investigation, 38, 1339-1348, 1959.

[22] ASCE: American Society of Civil Engineers. The ASCE Standardized reference evapotranspiration equation. In: Final Report of Environmental and Water Resources Institute of The American Society of Civil Engineers, 2005.

[23] American Society of Heating, Refrigerating and AirConditioning Engineers, Physiological principles and thermal comfort (ASHRAE). In: Handbook of Fundamentals, Atlanta, 2009.

[24] Bärtsch, P., Saltin, B. General introduction to altitude adaptation and mountain sickness. Scandinavian $J$. Medicine \& Science in Sports, 18, 1-10, 2008. 